\title{
Classification of perinatal deaths according to ICD-PM: An audit on perinatal post-mortems in a tertiary care centre in Sri Lanka
}

\author{
Priyani $\mathrm{AAH}^{\mathrm{a}}$, Thuvarakan $\mathrm{P}^{\mathrm{b}}$, de Silva MVC ${ }^{\mathrm{c}}$
}

\begin{abstract}
Introduction: Ending preventable perinatal deaths have become a priority in global public health agenda, highlighting the importance of collecting quality data related to perinatal mortality. Pathological post-mortems and placental examination play a vital role in identifying the cause of death. The objective of this analysis was to classify causes of death according to a uniform classification system, enabling comparison of data in different settings. Congenital malformations were further categorized according to the involved organ system.
\end{abstract}

Method: This is a retrospective analysis of post-mortems of stillbirths (including the placentas) and neonatal deaths already reported at the Department of Pathology, University of Colombo from 2009-2015. Autopsy and placental examination were performed according to standard protocols. The cause of death was classified according to ICD-PM.

Results: The study included 291 post-mortems; 198 stillbirths, 7 intra-partum deaths and 86 neonatal deaths. Ante-partum hypoxia was the commonest cause of death in stillbirths $(83 / 198=41.9 \%)$, while, congenital malformations was the commonest in intra-partum $(5 / 7=71.4 \%)$ and neonatal deaths $(38 / 86=44.2 \%)$. Low birth weight and prematurity is the second most common cause in neonatal deaths $(24 / 86=27.9 \%)$. Malformations were commonly present in cardiovascular system (stillbirths: $22 / 47=47 \%$, intra-partum and neonatal deaths: $31 / 62=50 \%$ ) followed by respiratory system (stillbirths: $14 / 47=30 \%$, intra-partum and neonatal deaths: $20 / 62=32 \%$ ).

Conclusion: Ante-partum hypoxia, congenital malformations and low birth weight and prematurity are the leading causes of death in stillbirths and neonates however, quality data of a prospective study, analyzing maternal and feto/infant pathology together, is required to improve the validity of the results.

Key words: Classification of perinatal deaths, ICD-PM, post-mortem

\section{INTRODUCTION}

Despite a decline in childhood mortality in last few decades, there is no significant

${ }^{a}$ Senior Lecturer, Department of Pathology,

Faculty of Medicine, University of Colombo

${ }^{b}$ Research Assistant, Department of Pathology,

Faculty of Medicine, University of Colombo

'Professor of Pathology, Department of

Pathology, Faculty of Medicine, University of

Colombo

Correspondence: Dr. AAH Priyani, MBBS,

D Path, MD (Pathology) Histopathology

Email:priyani@path.cmb.ac.lk

(D) http://orcid.org/0000-0003-4949-6265

Competing interests: The authors report no conflict of interest change in the perinatal mortality rates ${ }^{1}$. More than 5 million perinatal deaths are occurring globally each year ${ }^{2,3}$. Therefore, ending preventable stillbirths and neonatal deaths have become a priority in international agenda for public health, beyond $2015^{4}$, and there is an increased demand on medical and epidemiological grounds for proper determination and classification of causes of perinatal mortality, identification of cause of deaths and factors contributed to death. Majority of stillbirths (98\%) occur in low-income and middle-income countries; $77 \%$ in south Asia and sub-Saharan Africa ${ }^{5}$.
However quality data as to the cause of death and the conditions associated with high perinatal mortality are lacking from these regions, including Sri Lanka.

Autopsy and placental examination play a pivotal role in identification of cause of death and the underlying factors contributing to death, which is important not only for population based interventions but also for counselling parents and managing their subsequent pregnancies. Therefore, better understanding of pathology is of paramount importance for preventing the same adverse outcome happening in subsequent pregnancies.

Classification of causes of perinatal death is complex due to complicated pathophysiological processes occurring in the mother, fetus and placenta. More than thirty classification systems for perinatal mortality have been introduced since $1954^{6}$, which have been designed for different reasons with different approaches, definitions and levels of complexity, highlighting the need for a unifying globally acceptable classification system $^{6}$. Using the coding rules of the $10^{\text {th }}$ revision of the International Classification of Diseases and related health problems (ICD-10), World Health Organization (WHO), in collaboration with partners, has developed the WHO application of ICD-10 to perinatal deaths: ICD-perinatal mortality (ICD-PM) ${ }^{7}$. First, the perinatal deaths are classified according to the time of occurrence, as ante-partum, intrapartum or neonatal deaths. Then, ICDPM applies a multi-layered approach to the classification of cause of death, reflecting varying levels of available information depending on the setting. ICD-PM approach for classification of cause of death allows investigations such as post-mortem or placental histology to be captured, in settings where they are available ${ }^{7}$ Therefore, the ICD-PM, the current global approach in classification 
of perinatal deaths has recognized the place of post-mortem and the placental pathology in the identification of cause of death even for population based statistics.

None of the perinatal death classifications systems is perfect; however ICD-PM adopts the features of many classification systems and allows applicability in all settings and international comparisons ${ }^{7}$. Information derived from this classification will be useful to drive interventions and allocate resources to reduce preventable stillbirths and neonatal deaths.

This paper gives data of a post-mortem based study, classifying causes of stillbirths, intra-partum deaths and neonatal deaths according to ICD-PM, and identifying the commonly occurring congenital malformations related to organ systems in stillbirths, intra-partum deaths and neonatal deaths, and their associated risk factors.

\section{METHODOLOGY}

This is a retrospective analysis done at the Department of Pathology, University of Colombo during the period from 20092015. The already reported post-mortems of stillbirths (including the placentas), intra-partum deaths and neonatal deaths with sufficient clinical information were included in the analysis. Intrauterine deaths at or above 28 weeks of gestation were considered as stillbirths/ante-partum deaths. Deaths during the process of labour / caesarean section were considered intra-partum deaths. Live births after the gestation of 24 weeks or with a birth weight of $500 \mathrm{~g}$ or more and died within 1 month of life were considered as neonatal deaths. Neonates delivered before the period of gestation of 37 weeks were considered preterm.

The deaths for which only the placenta has been submitted for pathological examination were excluded from the study.

The details were extracted from the autopsy request forms, which give a summary of the antenatal follow-up records and the bed head tickets of the mother and the baby (in case of a neonate). These included, the maternal age, past medical history, parity, past obstetrics history, history of the present pregnancy, gestational age (at the time of death, in case of an intrauterine death and at the time of birth in case of a neonatal death), details of delivery and the neonatal care given (in case of a neonatal death).

Gross examination of the fetus/ neonate and placenta and the histological examination of the tissue were done according to standard protocols specified by the first.

Systematic external and internal examination of the fetus were done according to the standard procedure for perinatal autopsy ${ }^{9}$. Growth parameters were compared with standards growth charts ${ }^{10}$.

Histology sections were taken from all abnormal lesions and at least one section from liver, heart, lungs, thymus, spleen, kidneys, adrenals and brain.

The presence of congenital malformations were looked for and recorded in detail.

Gross and microscopic examination of the placentas including the umbilical cords and membranes were done according to a standard procedure for placental examination $^{11}$.

The congenital malformations were categorized according to the affected organ system and causes of death were classified according to ICD-PM assigning only one cause of death for each fetus/ neonate ${ }^{12}$.

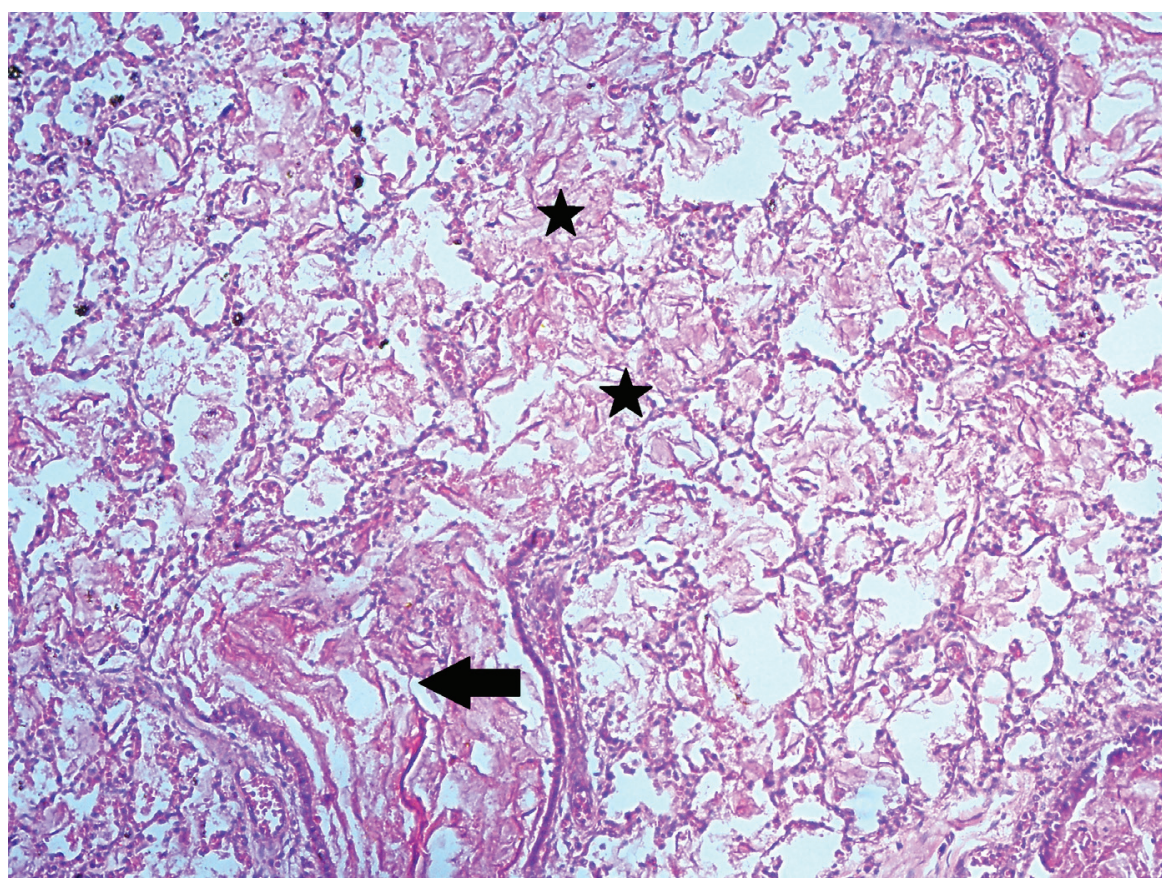

Figure 1: Numerous anucleate squames (stars) in the alveoli due to gasping of amniotic fluid contaminated with meconium. Meconium plugs in bronchioles are also seen (arrow) (H \& E X 200)

\section{RESULTS}

291 autopsies were included in this study, 198 were stillbirths with placentas and 93 were neonatal deaths. Out of 198 stillbirths, placentas were available in 157 cases. Out of 93 neonatal deaths, seven were intra-partum deaths registered as neonatal as resuscitation of the baby has been done despite very low Apgar scores at birth.

The maternal age of the study group ranged from 17 to 45 years with an average of 28.35 years.

$47 \%$ were primi-para and $25 \%, 18 \%$, $7.3 \%$ and $2 \%$ were the $2^{\text {nd }}, 3^{\text {rd }}, 4^{\text {th }}$ or $5^{\text {th }}$ para respectively.

The ICD-PM classification for cause of death for stillbirths, intra-partum deaths and neonatal deaths are shown in table 1. Ante-partum hypoxia was the commonest cause of death in stillbirths $(83 / 198=41.9 \%) \quad$ (Figure 1). Infection contributed only to $4 \%(n=8)$ of deaths (Figure 2). Congenital malformations contributed to deaths in $14 \%(28 / 198)$ stillbirths.

Congenital malformations were the commonest cause of death in intra-partum deaths $(5 / 7=71.4 \%)$ and the neonatal deaths $(38 / 86=44.2 \%)$. Low birth weight 


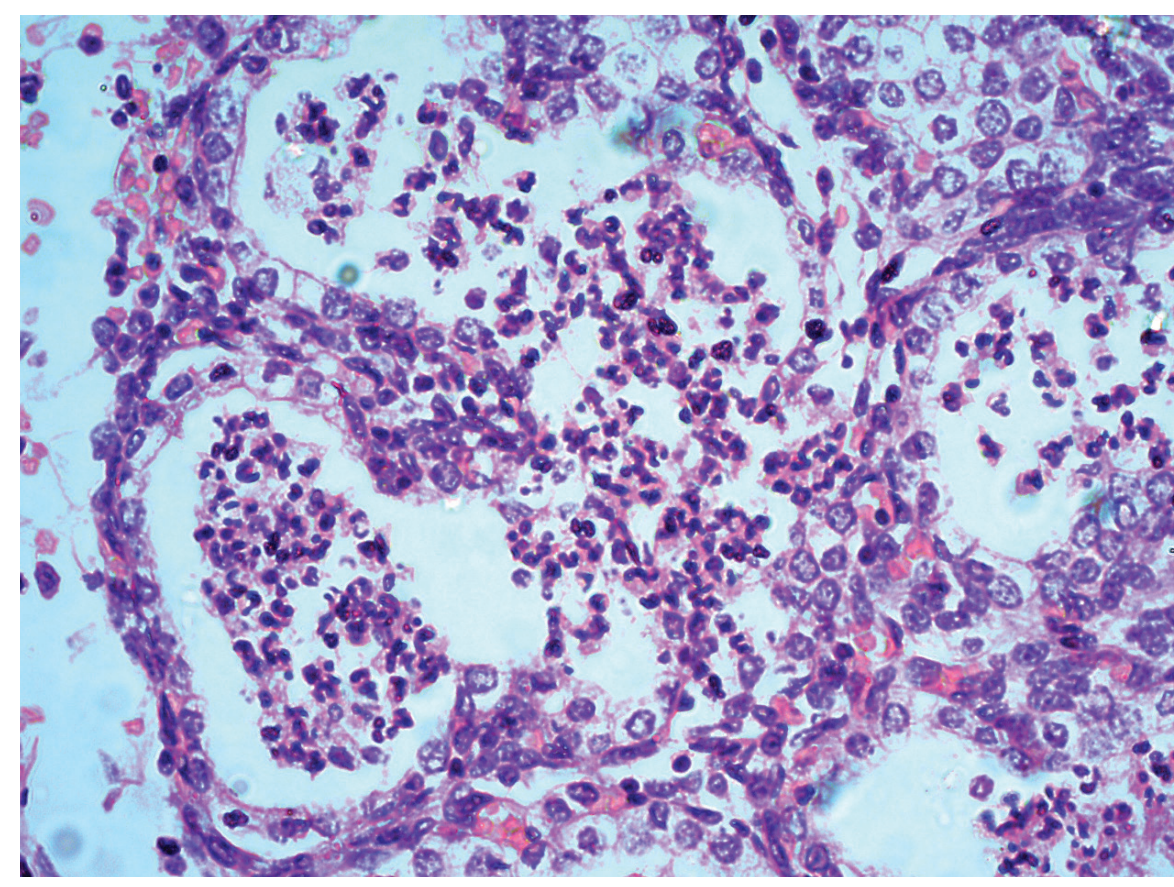

Figure 2: Neutrophils in alveolar spaces in the lungs in fetal pneumonia (H \& E X 400)

and prematurity was present in $27.9 \%$ $(24 / 86)$ of neonatal deaths.

Most of the congenital malformations were noted in the cardiovascular system followed by the respiratory system. Musculoskeletal and genitourinary system malformations were also relatively common both in stillbirths and in neonatal deaths (Table 2)

Cardiovascular malformations were noted in 53 babies, 36 had septal defects, eight had hypoplastic left heart and four had hypoplastic right heart. Pulmonary hypoplasia was the commonest respiratory system malformation, noted in 32 babies. Cleft lip was present in 10 and cleft palate was present in 11 babies. Genitourinary tract anomalies were present in 28 babies, 10 had obstructive defects, nine had renal agenesis and nine had cystic renal disease. The commonest musculoskeletal anomaly noted was deformities of foot, affecting 16 babies followed by diaphragmatic hernia affecting seven.

In $103(35.4 \%)$ autopsies, there was a history of maternal medical/ surgical conditions, diabetes mellitus in $15.1 \%$ (44), hypertension in $13.1 \%$ (38), heart disease in $6.2 \%(18)$, renal diseases/ SLE in $1.0 \%$ (3), thyroid disease in $1 \%$ (3) and rhesus incompatibility in $2.7 \%$ (8).

\section{DISCUSSION}

According to the analysis of cause of death in stillbirths and neonatal deaths in this setting, ante-partum hypoxia was the commonest cause of death in stillbirths whereas in intra-partum deaths and neonates, it was the congenital malformations. The second leading cause for neonatal deaths was low birth weight and prematurity. Deaths due to acute intrapartum events were less in this setting.

However, unlike other pathological conditions, categorizing ante-partum hypoxia as a cause of death is dificullt morphologically and should be done considering the clinical parameters. Histological features suggestive of tissue hypoxia include presence of numerous squames in alveolar spaces, increased number of nucleated red blood cells in fetal vessels, hypoxic injury of neurones and haemorrhage in internal organs, most of these are non-specific features. Further, ante-partum hypoxia is a condition which may result due to diverse pathologies both in the mother and the baby. Another drawback in this analysis is assessment of fetal growth (ICD code A5, I16, N5: Table 1) by one time reading of growth parameters that does not reflect the growth velocity of the baby during intra-uterine life. Lack of early dating scans also contributes to inacurrate assessment of fetal growth and maturity in this analysis.
Despite varying systems used so far for classification of perinatal deaths, some degree of comparison of this study with literature can be done as ICD-PM also has adopted the features of many other classification systems. WHO multi-center study was done in developing countries involving regularly followed up patients for calcium gluconate trial, which has shown that preterm birth (60.5\%), congenital malformations $(12.7 \%)$ and birth asphyxia $(22.5 \%)$ as main causes of death in newborns worldwide ${ }^{1}$. Contribution of congenital malformations as a cause of neonatal death is much less in this study compared to our setting which is $44 \%$.

According to a study done at neonatal intensive care unit in Alexandria, hyaline membrane disease was the leading cause of neonatal death (16.5\%) followed by other causes related to prematurity (15.8\%). Hyaline membrane disease was also the leading cause of death of preterm neonates $(27.5 \%)$. Birth asphyxia and congenital anomalies were the commonest causes of death among full term neonates $(21.7 \% \text { each })^{13}$. While the contribution by complications related to prematurity remains almost the same, the deaths due to congenital malformations are much higher (44\%) in our setting.

Comparison of data in different studies is difficult as the study populations are different. However, according to many studies done worldwide, preterm birth and complications related to prematurity, asphyxia and birth trauma, infections and congenital malformations were the leading causes of perinatal mortality ${ }^{1,13}$. The burden of conceptions with birth defects was underestimated according to the studies done in many settings compared to our study. This could be related to the option of termination of pregnancy available for the conceptions with lethal defects or because the statistics are not backed up by post-mortem examination of the baby. In Sri Lankan setting the conceptions with birth defects, unless terminated as spontaneous miscarriages, continue till birth adding a substantial proportion to the perinatal morbidity and mortality.

According to a ten-year study in Netherlands, the overall incidence of congenital malformations in the perinatal death-group was $33 \%{ }^{14}$. Through detailed post-mortem and placental examination 
Table 1- Classification of cause of death in stillbirths, intra-partum deaths and in neonatal deaths according to ICD-PM

\section{Stillbirths}

ICD code Classification of ante-partum deaths according to ICD PM

A1 Congenital malformations and chromosomal abnormalities

A2 Infection

A3 Ante-partum hypoxia

A4 Other specified ante-partum disorder

A5 Disorders related to fetal growth

A6 Fetal death of unspecified cause - Unable to classify

\section{Intra-partum deaths}

ICD code Classification of intra-partum deaths according to ICD PM Frequency

I1 Congenital malformations and chromosomal abnormalities

I2 Birth Trauma

I3 Acute intra-partum event

I4 Infection

I5 Other specified intra- partum disorder

I6 Disorders related to fetal growth

I7 Intra-partum death of unspecified cause - Unable to classify

\section{Neonatal deaths}

ICD code Classification of neonatal deaths according to ICD PM

$\begin{array}{ll}\text { N1 } & \text { Congenital malformations and chromosomal abnormalities } \\ \text { N2 } & \text { Disorders related to fetal growth } \\ \text { N3 } & \text { Birth trauma } \\ \text { N4 } & \text { Complications of intra-partum events } \\ \text { N5 } & \text { Convulsions and disorders of cerebral status } \\ \text { N6 } & \text { Infection } \\ \text { N7 } & \text { Respiratory \& cardiovascular disorders } \\ \text { N8 } & \text { Other neonatal conditions } \\ \text { N9 } & \text { Low birth weight and prematurity } \\ \text { N10 } & \text { Miscellaneous } \\ \text { N11 } & \text { Neonatal death of unspecified cause Unable to classify }\end{array}$

$5(71.4 \%)$ 0

$1(14.2 \%)$

0

0

$(14.2 \%)$

0
$8(4.0 \%)$

$83(41.9 \%)$

$35(17.7 \%)$

$10(5.1 \%)$

$34(17.2 \%)$
$28(14.1 \%)$ 0 $11(12.8 \%)$ 0

and clinical-pathological correlations, lethal congenital malformations were found in $51 \%$ of stillbirths (mainly central nervous system), and $70 \%$ of neonates (mainly cardiovascular and pulmonary defects $)^{14}$. The incidence of central nervous system malformations was low in the present study.

According to a study done in Bombay, malformation rates for live births, neonatal deaths, stillbirths and total births were, $1.02 \%, 9.24 \%, 9.49 \%$, and $1.40 \%$ respectively ${ }^{15}$. The musculoskeletal and central nervous system malformations were the commonest in this study ${ }^{15}$. However, musculoskeletal anomalies as a cause of death, was much less in our setting, while cardiovascular and respiratory anomalies were the leading cause of death.

\section{CONCLUSIONS}

Post-mortem examination of stillbirths and neonatal deaths and placental examination have identified leading causes of perinatal Frequency deaths in this study setting. However, $(\mathbf{n}=\mathbf{8 6}) \quad$ ICD-PM classification of pernatal deaths $38(44.2 \%)$ require analysis of the entire scenario $3(3.5 \%) \quad$ including obstetrics, neonatal and

Table 2 -Categorization of congenital malformations according to the involved organ system

$\begin{array}{lll}\text { Malformation } & \text { Stillbirths }(\mathbf{n}=\mathbf{4 7}) & \begin{array}{l}\text { Intra-partum and } \\ \text { Neonatal deaths }(\mathbf{n}=62)\end{array} \\ \text { CVS malformations } & 22(46.8 \%) & 31(50.0 \%) \\ \text { Respiratory system malformations } & 14(29.7 \%) & 20(32.2 \%) \\ \text { Musculoskeletal malformations } & 11(23.4 \%) & 18(29.0 \%) \\ \text { GUT malformations } & 9(19.1 \%) & 19(30.6 \%) \\ \text { CNS malformations } & 9(19.1 \%) & 13(20.9 \%) \\ \text { GIT malformations } & 4(08.5 \%) & 9(14.4 \%)\end{array}$
pathlological parameters, hence a detailed prospective multicenter study with a high post-mortem rate would be able to provide quality information which can be generalizable and will be useful to plan for interventions and preventive actions.

\section{REFERENCES}

1. Ngoc NTN, Merialdi M, AbdelAleem H. et al. Causes of stillbirths and early neonatal deaths: data from 7993 pregnancies in six developing countries. Bulletin of the World Health Organization. September 2006, 84(9): 699-705.

2. Blencowe H, Stanton C, Chou D. et al. National, regional, and worldwide estimates of stillbirth rates in 2009 with trends since 1995: a systematic analysis. Lancet. 2011 Apr 16; 377(9774): 1319-30.

3. Liu L, Oza S, Hogan D, Perin J, Rudan I, Lawn JE, et al. Global, regional, and national causes of child mortality in 2000-13, with projections to inform post-2015 priorities: an updated systematic analysis. Lancet. 2015; 385(9966): 430-40.

4. World Health Organization. Every 
Newborn: an action plan to end preventable deaths. Geneva: 2014. Available at http://apps.who.int/ (accessed on 3.5.2016).

5. Blencowe H, Cousens S, Jassir FB. Et al. National, regional, and worldwide estimates of stillbirth rates in 2015, with trends from 2000: a systematic analysis Lancet Glob Health 2016; 4: e98-108.

6. Korteweg FJ, Gordijn SJ, Timmer A. et al. The Tulip classification of perinatal mortality: introduction and multidisciplinary inter-rater agreement. BJOG. 2006;113:393401.

7. Allanson E, Tunçalp O, Gardosi J. et al. Classifying causes of perinatal deaths. Bull World Health Organ 2016;94:79-79A | doi: http://dx.doi. org/10.2471/BLT.15.168047

8. Thomas C. Michels, Alvin Y. Second Trimester Pregnancy Loss. Am Fam Physician 2007;76:1341-6, 1347-8.
9. Siebert JR. Perinatal, fetal, and embryonic autopsy. In: Enid GilbertBarness, ed. Potter's Pathology of the Fetus, Infant and Child, 2nd ed. China: Mosby Elsevier, 2007:695740 .

10. Gilbert-Barness E, Debich-Spicer DE. Handbook of paediatric autopsy pathology. Totowa: Humana Press, 2005.

11. Boyd TK, Redline RW. Pathology of the placenta. In: Enid GilbertBarness, ed. Potter's Pathology of the Fetus, Infant and Child, 2nd ed. China: Mosby Elsevier. 2007: 645694.

12. ICD-10 Chapter XVI: Certain conditions originating in the perinatal period.available at https:// en.wikipedia.org/wiki/ (accessed on 2. 05.2016).

13. Fikry MM. Identification of causes of neonatal mortality using the ICD10 classification: a study in neonatal intensive care units in Alexandria
Governorate (MOHP). J Egypt Public Health Assoc. 2003; 78(1-2): 127-52.

14. De Galan-Roosen AE, Kuijpers JC, Meershoek AP, van Velzen D. Contribution of congenital malformations to perinatal mortality. A 10 years prospective regional study in The Netherlands. Eur J Obstet Gynecol Reprod Biol. 1998 Sep; 80(1): 55-61.

15. Kolah PJ, Master PA, Sanghvi LD. Congenital malformations and perinatal mortality in Bombay. Indian Cancer Research Centre, Parel Bombay, India. DOI: http://dx.doi. org/10.1016/0002-9378(67) 90505-4. 